\title{
Influences of preoperative metformin on immunological factors in early breast cancer
}

\author{
Takahiro Tsukioki $^{1} \cdot$ Tadahiko Shien $^{1}$ (1) $\cdot$ Takehiro Tanaka $^{2} \cdot$ Yoko Suzuki $^{1} \cdot$ Yukiko Kajihara $^{1} \cdot$ Minami Hatono $^{1}$. \\ Kengo Kawada ${ }^{1} \cdot$ Mariko Kochi $^{1} \cdot$ Takayuki Iwamoto $^{1} \cdot$ Hirokuni Ikeda $^{1} \cdot$ Naruto Taira ${ }^{1} \cdot$ Hiroyoshi Doihara $^{1}$. \\ Shinichi Toyooka'
}

Received: 16 February 2020 / Accepted: 3 June 2020 / Published online: 12 June 2020

(c) The Author(s) 2020

\begin{abstract}
Purpose Metformin has been suggested to possibly reduce cancer risk. However, the mechanism underlying the positive effects of metformin on cancer treatment remains unclear. We conducted a prospective study to evaluate the effects of preoperative metformin in patients with early breast cancer.

Method We evaluated the effects on immunological factors (TILs, CD4 +, CD8 +, PD-L1, IFN $\gamma$ and IL-2) by comparing core needle biopsies (CNB) obtained before metformin treatment with surgical specimens. Seventeen patients were enrolled in this prospective study from January to December 2016. We also analyzed 59 patients undergoing surgery during the same period to reveal the correlation of immune factors between CNB and surgical specimen.

Result There was a moderate correlation between CNB and surgical specimens on TILs and CD8 + lymphocyte. (TILs $\mathrm{Rs}=0.63, \mathrm{CD} 4+\mathrm{Rs}=0.224, \mathrm{CD} 8+\mathrm{Rs}=0.42)$ In the metformin group, TILs increases were confirmed in five (29\%) patients, while a decrease was confirmed in two (12\%). The expressions of CD4 + and CD8 + by TILs were increased in $41 \%$ and $18 \%$ of surgical specimens, respectively. However, TILs number $(p=0.0554)$, CD4+ $(p=0.0613)$ and CD8 $+(p=0.0646)$ expressions did not significantly increased. Furthermore, IFN $\gamma$ expression appeared to be increased in response to metformin $(p=0.08)$.

Conclusion Preoperative metformin tends to increase TILs, as well as the numbers of CD4 and CD8 positive lymphocytes, and IFN $\gamma$ levels. Metformin might improve immune function and have a possibility of chemo-sensitivity and thereby increase the effectiveness of immunotherapy, based on the results of this preliminary study.
\end{abstract}

Keywords Breast cancer · Metformin · Preoperative · Tils · CD8 · PD-L1

Electronic supplementary material The online version of this article (https://doi.org/10.1007/s00280-020-04092-2) contains supplementary material, which is available to authorized users.

Tadahiko Shien

tshien@md.okayama-u.ac.jp

1 Department of General Thoracic Surgery and Breast and Endocrine Surgery, Okayama University Graduate School of Medicine, Dentistry, and Pharmaceutical Sciences, 2-5-1 Shikata-cho, Kita-ku, Okayama 7008558, Japan

2 Department of Pathology, Okayama University Hospital, 2-5-1 Shikata-cho, Kita-ku, Okayama 700-8558, Japan

\section{Introduction}

Breast cancer is among the major causes of cancer-related death in women worldwide. While significant progress has been made in the diagnosis and treatment of breast cancer, the clinical outcomes of patients are still discouraging [1, 2]. Diabetes mellitus (DM) has an adverse impact on cancer. A systematic review indicated DM patients to have a $17 \%$ increased risk for breast cancer mortality [3]. One report has suggested that treating DM with metformin, that is one of the commonly prescribed drugs for type $2 \mathrm{DM}$, reduces the risk of breast cancer death as compared with sulfonylureas and insulin formulations [4]. However, the mechanism underlying the favorable effect of metformin on cancer epidemiology has yet to be investigated in detail. 
Preclinical research using both breast cancer cell lines and mouse models subsequently showed that metformin represses cancer cell and xenograft growth. Some of the reported mechanisms are that metformin preferentially kills cancer stem cells through activation of AMP-activated protein kinase, leading to activation of FOXP 3 , and the inhibition of m-TOR, a crucial signaling pathway for cellular proliferation and the survival of cancer cells, and also exerts an anti-tumor effect by enhancing immune function [5-7]. Of these mechanisms, the metformin's effect on tumor microenvironment including immune functions is drawing attentions. Eikawa et al. showed that an anti-tumor effect of metformin is dependent on CD8+ T-cells in a murine model [7]. Nicole et al. reported that although tumor hypoxia inhibits T-cell function, metformin improves an inhibitor of tumor oxygen consumption and increases activation of T-cell [8].

Moreover, it is certain that the interplay between immune cells and tumor cells exerts a major influence on both the development and the progression of breast cancer [9]. Especially, the role of the cytotoxic $\mathrm{T}$ lymphocytes in tumorigenesis, has been explored extensively. T-cell infiltration in invasive breast cancer has been reported that secrete several inflammatory cytokines, for example interferon- $\gamma$ (IFN $\gamma$ ), transforming growth factor beta (TGF- $\beta$ ), tumor necrosis factor alpha (TNF $\alpha$ ) and interleukin-2 (IL-2). These cytokines then interact with other cytotoxic T-cells and upregulate the MHC class I and II molecules, as well as other antigen display co-factors in neoplastic cells [10]. We believe this process is an essential part of immune-mediated anti-tumorigenic effects.

The immune factor has an important role in clinical practice. Tumor infiltrating lymphocytes (TILs) are known to be prognostic factors as well as markers predicting chemo-sensitivity $[11,12]$. Mao et al. conducted a meta-analysis which showed that higher levels of TILs correlated with the pCR rate in response to neoadjuvant chemotherapy (NAC) [15]. Other reports show that TILs is a significant predictor of the pathologic complete response in patients with triple-negative (TN) and human epidermal growth factor receptor-2 (HER2) positive breast cancer subtypes [13, 14]. In addition, various TIL subsets have different roles in breast cancer progression. $\mathrm{CD} 4+$ and $\mathrm{CD} 8+$ lymphocytes play crucial parts in determining cancer treatment and high intra-tumoral lymphocyte counts were found to be associated with better survival in breast cancer patients [16]. And higher levels of CD8 + T-lymphocytes in pre-treatment biopsy specimens predicted a better pathological response to NAC [15]. And cancer immunotherapy (PD-1, PDL-1 antigen) approaches have become among the most effective treatments for metastatic breast cancer [17].

Thus, we speculate that metformin improves immune function and favorably modifies the tumor environment. To our knowledge, there are no prior studies examining the influences of metformin on TILs, CD4 +, CD8 +, IFN $\gamma$, IL-2 and PDL-1 in patients without DM. We conducted this prospective study to confirm the effects of preoperative metformin in patients with early breast cancer (metformin study).

\section{Materials and methods}

To evaluate the effects of metformin, we decided to do two studies. First, we gathered patients who had been diagnosed with breast cancer by $\mathrm{CNB}$ and after that they took metformin in the preoperative period, and we compare CNB that was pre-metformin with surgical specimen that was post-metformin. (metformin study) Second, because of breast cancer is known to have tumor heterogeneities, it is not uncertain to compare CNB with surgical specimen on immune functions. So, we should analyze the correlations of immune functions between CNB and surgical specimens. We assessed patients who underwent surgery and did not take metformin during the same period. (correlation study).

The patients, who had newly diagnosed stage I or IIA breast cancer and no history or evidence of DM based on blood biochemistry, were not taking metformin before enrollment in this study. All participants were enrolled between January and December 2016 at Okayama University Hospital. All participants were then administered a daily dose of metformin orally for 2 weeks before surgery. All 17 patients had the same oral metformin dosing schedule: $500 \mathrm{mg}$ daily for 3 days $(250 \mathrm{mg}$ twice after the morning and evening meal), increased to $750 \mathrm{mg}$ daily for next 4 days ( $250 \mathrm{mg}$ three times after every meals), and if no adverse effects were observed, the dose was increased to $1000 \mathrm{mg}$ daily for next 7 days before surgery (500 mg twice after the morning and evening meal).

Patients who received NAC or had recurrent tumors were excluded. Hematoxylin-eosin (HE)-stained slides of CNB and matched surgical specimens were reviewed retrospectively. We evaluated the expressions of and concordance between immuno-histological factors in CNB and surgical specimens from the same patient.

All clinical data were retrospectively extracted from our institution's electronic medical records system. Metformin study was approved by the Ethics Committee of Okayama University Hospital (UMIN 000,014,090) and adhered to the Declaration of Helsinki. We obtained consent in writing from each patient. In addition, correlation study was approved by the Ethics Committee of Okayama University Hospital and adhered to the Declaration of Helsinki. 


\section{Immunohistochemistry}

Tumor morphology was evaluated using conventional HEstaining. Estrogen receptor (ER)/ progesterone receptor (PR) and HER2 were assessed according to standard guidelines. Nuclear staining $\geqq 1 \%$ for estrogen receptors (ER) or progesterone receptors (PgR) was considered to be positive. HER 2 positivity was defined as $3+$ receptor overexpression on immunohistochemical (IHC) staining or gene amplification on fluorescence hybridization using a HER2/CEP ratio $\geqq 2.0$. The expressions of ER, PgR, HER2 and the Ki67 labelling index (Ki67) in both CNB and surgical specimens were determined.

Prior to IHC staining for immune factors, the tumor specimens were fixed in $10 \%$ formaldehyde solution and embedded in paraffin, then sliced into $4-\mu \mathrm{m}$-thick sections. We employed the Ventana Discovery XT automated platform (Ventana Medical Systems: Roche Tissue Diagnostics). Primary monoclonal antibodies directed against CD4 (SP35 Rabbit monoclonal antibody; Roche Tissue Diagnostics), CD8 (SP57 Rabbit monoclonal antibody; Roche Tissue Diagnostics), IFN $\gamma$ (ab9657 Rabbit anti-interferon gamma antibody; Abcam), IL-2 (EPR2780 Rabbit monoclonal antibody; Abcam) and PD-L1 (22C3 Mouse monoclonal antihuman PD-L1 antibody; Dako North American) were used.

To determine the efficacy of metformin, we evaluated TILs, as well as CD4, CD8, IFN $\gamma$, IL-2 and PDL-1 expressions, in both CNB and surgical specimens (Fig. 1).

\section{Evaluation of TILs}

HE-stained sections were utilized for evaluation for TILs. The percentages of stromal lymphocytes, serving as a predefined criterion in Denkert's reports, were evaluated by one highly experienced observer [14, 18, 19]. Stromal TILs were measured as the percentage of immune cells in stromal tissue within the tumor that showed a mononuclear immunological infiltrate. Heterogeneous distributions were documented in nearly all sections. Therefore, hot spots, cold spots, and Tertiary Lymphoid Structure were not taken into consideration in conducting the measurements, with just one representative area being selected and evaluated. We divided the proportions of TILs into $10 \%$ increments. TILs were categorized according to three cut-off values, as a dichotomous variable (0 to $10 \%$ : negative, $11-30 \%$ : low, 31-50\%: intermediate, over 51\%: high). We also decided whether there was a $20 \%$ difference between the CNB and the surgical specimen.

\section{Evaluation of CD4 + and CD8 + T-lymphocytes, IFNy, IL-2 and PD-L1}

Based on previous studies, CD4 + and CD8 + expressions were calculated using the average of stained TILs in the area that was maximally stained, viewed at $400 \times$ magnification $[20,21]$. We randomly selected two different high-power fields and counted those expressing CD4 + or CD8 + in 100 lymphocytes.

The semi-quantitative $\mathrm{H}$ scoring system was employed, using whole tissue sections, to evaluate the presence of IFN $\gamma$ and IL-2. The $\mathrm{H}$ score was calculated by multiplying the percentage of positive cells (tumor and immune) by a factor representing the intensity of immune-reactivity ( 1 for weak, 2 for moderate, and 3 for strong), giving a maximum score of 300. A score below 50 was considered negative and a score of over 50 was positive.
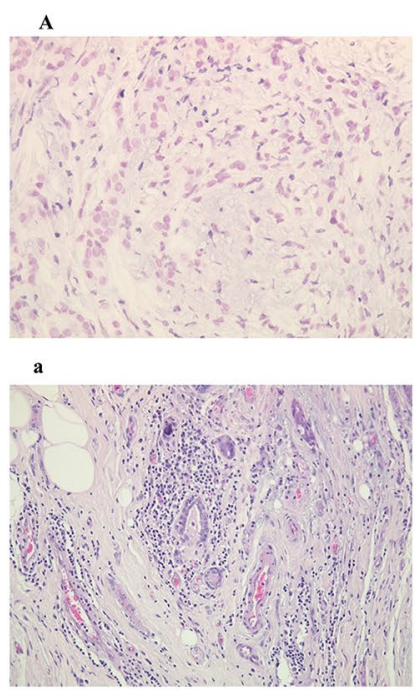

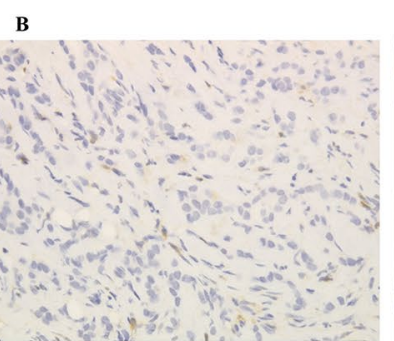

b

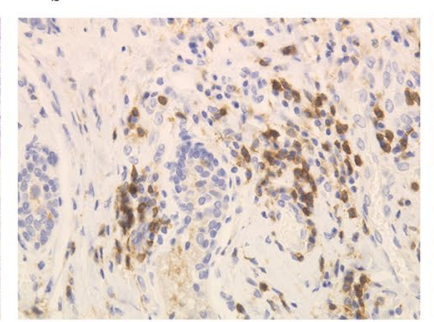

$\mathrm{C}$
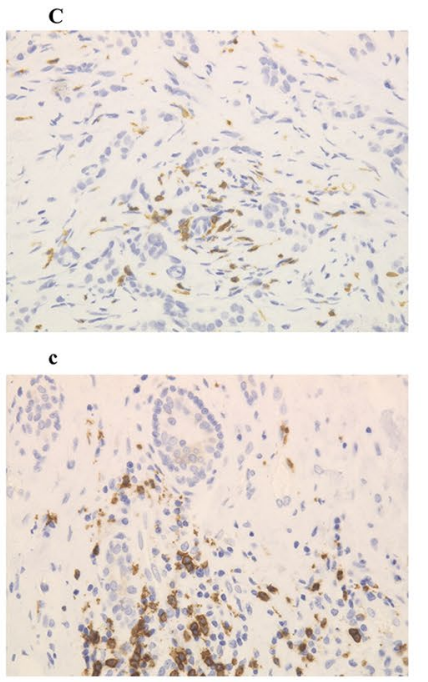

D
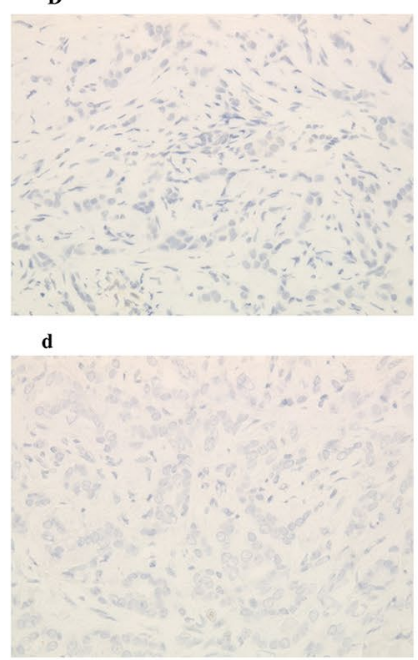

Fig. 1 The expression of TILs, CD4 +, CD8 + and PD-L1 both CNB and surgical specimens among the same patient. There was increasing of TILs, CD4 + and CD8 + lymphocyte. TILs: (A, a), CD4 + : (B, b), CD8 + lymphocytes: (C, c), PD-L1: (D, d) 
PD-L1 expression was categorized as positive when staining of the tumor-cell membrane (at any intensity) was observed at pre-specified expression levels of $1 \%, 5 \%$, or $10 \%$ of cells in a section that included at least 100 tumor cells that could be evaluated [17]. As judging PD-L1 expression is difficult, we asked an experienced pathologist to determine the expression rates.

\section{Statistical analysis}

This study was a non-planned, retrospective, exploratory project, for which all available cases were used without a predefined sample size calculation to detect a specific effect size or reach a certain level of power. Data were analyzed with EZR (version 1.37) [22]. $P$ values $<0.05$ were considered to indicate a statistically significant difference.

The immune related factors of CNB and surgical specimens were compared using Spearman's paired rank correlation coefficient (Rs). To evaluate the correlation between the TILs, CD4 + and CD8 + lymphocytes in CNB and surgical specimens, Rs was calculated. Rs of $<0.4,0.4-0.7,0.7-0.9$, and $>0.9$ indicated poor, moderate, good, and excellent reliability, respectively.

To evaluate and compare the effects of preoperative metformin, we defined changes in TILs, CD4 + and CD8 + lymphocytes exceeding $20 \%$ as positive. The Related-Samples Wilcoxon Signed Rank test was performed for comparisons of the expressions of all immune factors.

\section{Results}

\section{Correlation of TILs, CD4 + and CD8 + T-lymphocyte numbers between CNB and surgical specimens}

Fifty-nine patients were evaluated as a correlation group. Patient characteristics are presented in Table 1. The median age was 65 (32-87) years. The median tumor size was $15 \mathrm{~mm}$ (range 2-52). Invasive ductal carcinoma accounted for $56(95 \%)$ and invasive lobular carcinoma for $3(5 \%)$ cases. Histological grade was III in 22 (37\%), II in 7 (12\%) and $\mathrm{I}$ in $30(51 \%)$ cases. The Ki67 index was high (over $30 \%)$ in $22(37 \%)$, intermediate (15-29\%) in $17(29 \%)$ and low (under $14 \%)$ in 20 (34\%) cases. The breast cancer subtype was luminal A type $(\mathrm{ER}+, \mathrm{HER} 2-, \mathrm{Ki} 67<15 \%)$ in 19 $(32 \%)$, luminal B type $(\mathrm{ER}+, \mathrm{HER} 2-, \mathrm{Ki} 67>15 \%)$ in 23 (39\%), luminal HER2 type in 4 (7\%), HER2-enriched type in $4(7 \%)$ and TN type in $9(15 \%)$ cases (Table 1$)$. The status of common biological markers did not differ between CNB and surgical specimens (Supplementary Table 1).

TILs in CNB were negative in 37 (63\%), low in 18 (30\%), intermediate in $4(7 \%)$ and high in 0 cases. The corresponding values in surgical specimens were 34 (58\%), 16 (27\%),
Table 1 Clinicopathological characteristics of the correlation study group $(n=59)$

\begin{tabular}{ll}
\hline & Values \\
\hline Age & \\
Median (range) & $65(32-87)$ \\
Distribution—no. (\%) & \\
$>50$ & $13(22)$ \\
$\leq 50$ & $46(78)$ \\
Tumor size-median (range: mm) & $15(2-52)$ \\
T1a-no. (\%) & $3(5)$ \\
T1b & $15(25)$ \\
T1c & $22(37)$ \\
T2 & $18(31)$ \\
T3.T4 & $1(2)$ \\
Tumor grade (surgical specimen)—no. (\%) & \\
I & $30(51)$ \\
II & $7(12)$ \\
III & $22(37)$ \\
Ki67 index—no. (\%) & \\
0-14 & $20(34)$ \\
15-29 & $17(29)$ \\
30 & $22(37)$ \\
Lymph node metastasis—no. (\%) & \\
Positive & $12(20)$ \\
Negative & $47(80)$ \\
Lubtype (surgical specimen)—no. (\%) & \\
Luminal A & $19(32)$ \\
Luminal B (HER2 positive) & $23(39)$ \\
HER2-enriched type & $4(7)$ \\
Triple negative & $4(7)$ \\
\hline & $9(15)$ \\
\hline
\end{tabular}

$4(7 \%)$ and $5(8 \%)$. The median numbers of lymphocytes expressing CD4 + and CD8 + were 34 and 27, respectively, in CNB and surgical specimens. We also evaluated 20 intervals of the surgical specimen scores, yielding CD4 + numbers of 17 (29\%: 0 to 20), 24 (41\%: 21 to 40) and 18 (30\%: over 41) for CNB and CD8 + numbers of $19(32 \%), 30(51 \%)$ and $10(17 \%)$, respectively, for surgical specimens. (Supplementary Table 1) We attributed this discordance to a difference in the numbers of lymphocytes exceeding $20 \%$ between the CNB and surgical specimens. The total concordance rates, no difference, of the levels of CD4+ and CD8 + lymphocytes were 25 (43\%) and 29 (49\%), respectively (Supplementary Table 2).

Correlation analysis showed moderate positive TILs correlations between $\mathrm{CNB}$ and resected specimens $(\mathrm{Rs}=0.63$, $p=0.0000000872$ ). We evaluated tumor size, tumor grade, subtype and Ki67 index results. All subgroup analyses showed positive correlations. (data not shown) Most notably, tumor size, Grade III, HER2-enriched type and a high Ki67 
index tended to show significant positive correlations (the Rs of these factors were all over 0.7 ). For TIL subsets, correlations with $\mathrm{CD} 4+$ were poor $(\mathrm{Rs}=0.224, p=0.0875)$, while moderate correlations with $\mathrm{CD} 8+$ were noted $(\mathrm{Rs}=0.42, p$ $=0.00917)($ Table 2$)$.

\section{Distributions of and changes in TILs, CD4 + and CD8 + lymphocytes in CNB and surgical specimens from patients receiving metformin preoperatively}

Seventeen patients were enrolled in the prospective metformin study. Patient characteristics are presented in Table 3. The median age was 58 (36-74). The median tumor size was $13 \mathrm{~mm}(2-23)$. Histological grade was III in six (35\%), II in six (35\%) and I in five (30\%) cases. Nine patients had lymphatic invasion, but none had evidence of vascular invasion. The Ki67 index was high (over 30) in $6(35 \%)$, intermediate (15-29) in $6(35 \%)$ and low (under 14) in $5(30 \%)$ cases. The breast cancer subtype was luminal A type (ER +, HER2-, Ki67 < 15\%) in six (35\%), luminal B type (ER +, HER2-, Ki67 > 15\%) in five (30\%), luminal HER2 type in one $(6 \%)$ and TN type in five (30\%) cases. The CNB specimens obtained before metformin and the surgical specimens did not differ in terms of biological markers. Five patients (29\%) were lymph node-positive. Nearly all characteristics and pathological biomarkers were differed minimally between the metformin and correlation groups (Table 3).

Table 2 Correlations between CNB and surgical specimens, compared using the paired Spearman's rank correlation coefficient (Rs), in the correlation study group

\begin{tabular}{lll}
\hline & Spearman's Rs & $P$ value \\
\hline All patients & $r=0.63$ & $p<0.001$ \\
Tumor size & & \\
T1 $(n=40)$ & $r=0.58$ & $p<0.001$ \\
T2,3,4 $(n=19)$ & $r=0.745$ & $p<0.001$ \\
Tumor Grade & & \\
I ( $n=30)$ & $r=0.469$ & $p=0.00898$ \\
II $(n=7)$ & $r=0.558$ & $p=0.193$ \\
III $(n=22)$ & $r=0.789$ & $p<0.001$ \\
Ki67 index & & \\
$<30 \%(n=37)$ & $r=0.431$ & $p=0.00772$ \\
$\geq 30$ ( $n=22)$ & $r=0.765$ & $p<0.001$ \\
Subtype & & \\
Luminal A & $r=0.424$ & $p=0.00465$ \\
Luminal B (HER2 negative) & $r=0.327$ & $p=0.16$ \\
Luminal B (HER2 positive) & $r=0.531$ & $p=0.0097$ \\
HER2-enriched type & $r=0.937$ & $p<0.001$ \\
Triple negative & $r=0.687$ & $p=0.00409$ \\
CD4 + lymphocytes & $r=0.224$ & $p=0.0875$ \\
CD8 + lymphocytes & $r=0.42$ & $p=0.00917$ \\
\hline
\end{tabular}

Table 3 Clinicopathological characteristics and tumor biology of the metformin study group $(n=17)$

\begin{tabular}{ll}
\hline & Values \\
\hline Age & \\
Median (range) & $58(36-74)$ \\
Distribution—no. (\%) & \\
$>50$ & $4(24)$ \\
$\leq 50$ & $13(76)$ \\
Tumor size-median (range: mm) & $13(2-23)$ \\
T1a-no. (\%) & $4(24)$ \\
T1b & $2(12)$ \\
T1c & $10(59)$ \\
T2 & $1(6)$ \\
T3.T4 & $0(0)$ \\
Tumor Grade (surgical specimen)—no. (\%) & \\
I & $5(30)$ \\
II & $6(35)$ \\
III & $6(35)$ \\
Ki67 index—no. (\%) & \\
$0-14$ & $5(30)$ \\
15-29 & $6(35)$ \\
30 & $6(35)$ \\
Lymph node metastasis—no. (\%) & \\
Positive & $5(29)$ \\
Negative & $12(71)$ \\
Lubtype (surgical specimen)—no. (\%) & \\
Luminal B (HER2 negative) & $6(35)$ \\
Luminal B (HER2 positive) & $5(30)$ \\
HER2-enriched type & $1(6)$ \\
Triple negative & $0(0)$ \\
\hline & $5(30)$ \\
\hline
\end{tabular}

Fifteen patients were able to take metformin for 2 weeks, as planned. Two patients experienced nausea (GradeI) 3 days after starting metformin, and therefore discontinued this medication.

The distributions of TILs, CD4 + and CD8 + lymphocytes are shown in Table 4. The TIL expression results before taking metformin were negative in $15(89 \%)$ and low in 2 (11\%) patients, while after metformin $8(47 \%)$ cases were negative, $8(47 \%)$ had low and $1(6 \%)$ showed intermediate expression. And CD4+lymphocytes expression was that 5 (29\%) patients were over $20 \%$, while after metformin there were $12(71 \%)$. CD8 + lymphocytes expression was that 6 $(35 \%)$ were over $20 \%$, while after metformin there were 10 (59\%) (Table 4).

We determined the percentages of TILs expressing $\mathrm{CD} 4+$ and $\mathrm{CD} 8+$. We defined a change of at least $20 \%$ as indicating metformin efficacy. Comparison of the results obtained before versus after metformin showed an increase in seven $(42 \%)$ cases, while one $(6 \%)$ showed a decrease 
Table 4 Changes of immune-related factors expression before and after metformin administration

\begin{tabular}{lll}
\hline & $\begin{array}{l}\text { Pre-metformin: } \\
\text { CNB-no. } \%)\end{array}$ & $\begin{array}{l}\text { Post-metformin: } \\
\text { surgical-no. } \\
(\%)\end{array}$ \\
\hline TILs & & \\
Negative:0-10\% & $15(89)$ & $8(47)$ \\
Low:11-30\% & $2(11)$ & $8(47)$ \\
Intermediate:31-50\% & $0(0)$ & $1(6)$ \\
High:>50\% & $0(0)$ & $0(0)$ \\
CD4+lymphocytes & & $5(29)$ \\
$0-20 \%$ & $12(70)$ & $7(41)$ \\
$21-40 \%$ & $3(17)$ & $4(24)$ \\
$41-60 \%$ & $1(6)$ & $1(6)$ \\
$>60 \%$ & $1(6)$ & $7(41)$ \\
CD8+lymphocytes & & $10(59)$ \\
$0-20 \%$ & $11(65)$ & $0(0)$ \\
$21-40 \%$ & $6(35)$ & $0(0)$ \\
$41-60 \%$ & $0(0)$ & $6(35)$ \\
$>60 \%$ & $0(0)$ & $2(12)$ \\
IFN $\gamma$ positive: H score $\geq 50$ & $2(15)$ & $7(41)$ \\
IL-2 positive: H score $\geq 50$ & $1(6)$ & $4(24)$ \\
PD-L1 positive & &
\end{tabular}

Table 5 Statistical analysis of the preoperative metformin influence, compared using the Related-Samples Wilcoxon Signed Rank test

\begin{tabular}{llll}
\hline & $\begin{array}{l}\text { Increasing- } \\
\text { no. }(\%)\end{array}$ & $\begin{array}{l}\text { No increas- } \\
\text { ing-no. }(\%)\end{array}$ & $P$ value \\
\hline TILs & $7(42)$ & $10(58)$ & $p=0.0545$ \\
CD4 + lymphocytes & $9(53)$ & $8(48)$ & $p=0.0613$ \\
CD8 + lymphocytes & $6(35)$ & $11(65)$ & $p=0.0646$ \\
IFN & $5(38)$ & $8(62)$ & $p=0.0803$ \\
IL-2 & $6(35)$ & $11(65)$ & $p=1$ \\
PD-L1 & $3(18)$ & $14(82)$ & $p=0.572$ \\
\hline
\end{tabular}

in TILs. 9 cases (53\%) had increased CD4+, while four (24\%) showed a decrease. Six cases (35\%) had increased $\mathrm{CD} 8+$ and one $(6 \%)$ showed a decrease (Supplementary Table 3).

But there were no significant differences in the change of TILs, CD $4+$ and CD $8+$ lymphocytes, comparing the patients increasing more than $20 \%$ or not (Table 5).

\section{Distributions of and changes in IFNy, IL-2 and PD-L1 in CNB and surgical specimens from patients receiving metformin preoperatively}

We judged IFN $\gamma$ and IL-2 expressions by the $\mathrm{H}$ score. IFN $\gamma$ expression in CNB was positive in two cases (15\%) preoperatively, while six (35\%) cases were positive after metformin administration. IL-2 expression in $\mathrm{CNB}$ was positive in one case $(6 \%)$ preoperatively, while two $(12 \%)$ were positive after taking metformin.

PD-L1 expression was judged as being negative (0\%), low (1-9\%) or high $(\geq 10 \%)$. Thirteen $(76 \%)$ cases were negative, two (12\%) had low and two (12\%) had high PD-L1 expression in CNB. The corresponding values in surgical specimens were ten (59\%), five (30\%) and two (12\%) (Table 4).

Comparisons of the CNB and surgical specimens yielded no statistically significant differences in the rises in the levels of IFN $\gamma(p=0.0803)$, IL-2 $(p=1)$ and PD-L1 $(p=0.572)$ (Table 5).

\section{Discussion}

Interpreting limited samples, such as $\mathrm{CNB}$, raises questions about both sampling adequacy and error. Regarding the points, the extent and potential effects of sample type and the spatial heterogeneity of tumor immune infiltrates have yet to be examined in sufficient detail. The clinical and scientific importance of assessing TILs in breast cancer has been highlighted by recent efforts to standardize the histologic interpretation of TILs in patient samples. However, neither TIL heterogeneity nor the adequacy of core biopsy samples versus tissue sections has been fully documented [23]. This difference might be attributable to the limited area of tumor tissue biopsied, with indefinite tumor borders, in the CNB. However, Cha et al. reported there was less than $5 \%$ differences between TILs of CNB and surgical specimen [24]. Compared with their study, there was slightly differences of TILs between CNB and surgical specimen in our study, but Rs showed moderately positive correlations. (Rs $=0.63)$ Thus, we concluded that TILs in CNB specimens constitute a reliable indicator of the TIL values for the entire surgically resected breast tumor. In addition, in clinical settings, sequential tumor core biopsies have become accepted in NAC and window-of-opportunity studies as a means of assessing early evidence of the therapeutic efficacy of an agent or a treatment strategy [25-27]. These have included neoadjuvant endocrine trials $[28,29]$ and the use of novel agents [27] in window-of-opportunity studies. These trials have identified the Ki67 index at 2 weeks as a predictor of relapse free survival [28] or efficacy [30] and as a prognostic marker for adjuvant chemotherapy $[31,32]$. Therefore, it is important to determine whether CNB reflects the biology of the entire tumor.

The mechanism underlying the effects of metformin on the cancer remains uncertain. In chronic infectious diseases and cancer, CD8 + T cells specific for viral and/ or tumor antigens undergo repeated TCR stimulation due to the presence of persistent pathogens or cancer cells and gradually lose their ability to secrete IL-2, TNF $\alpha$, 
and IFN $\gamma$, eventually undergoing apoptotic elimination in a process known as immune exhaustion [33]. Eikawa revealed that metformin prevents apoptosis of CD8 + TILs and induces multifunctional CD8 + effector memory T-cells expressing the exhaustion marker Tim-3 in an in vivo murine study [7]. Furthermore, cancer immunotherapy (PD-1, PDL-1 antigen) has now been recognized as being among the most effective strategies for treating lung cancer and melanoma. In metastatic breast cancer, immunotherapy yields favorable outcomes. Immunotherapy efficacy may depend on TILs. If so, developing methods of increasing TILs may become an increasingly important research goal.

Since 1957, immuno-editing has been identified in a wide range of tumor progression forms. However, the relationships between tumors and factors comprising the immune system remain complex and still are not fully understood. Yan et al. reported that TILs with CD8 + are the main effector cells in the immune response, being associated with better disease-free survivals, but not improved overall survivals. CD4 + lymphocytes are composed of both $\mathrm{T}$ helper and regulatory cells, such that their roles are highly complex. Furthermore, according to Mao Y et al.'s meta-analysis, CD4 + lymphocytes are not prognostic markers for breast cancer [15]. However, CD4 + data are very limited, and more prospective studies are needed to confirm their prognostic value in breast cancer. According to these evidences, if metformin improves immune function and increases the number of TILs, CD4 + and CD8 + lymphocytes, preoperative metformin administration would be expected to enhance chemotherapy effects. We did not identify a statistically significant increase in TILs, CD4 + or CD8 + lymphocytes with preoperative metformin, but an increasing trend was noted.

These non-statistically significant results might be due to the metformin administration being short-term and low dose, to the small number of patients and to there being few large tumors and/or discordance among the breast cancer types. A previous randomized study examined the effects of metformin in non-DM patients. Ko et al. reported that patients taking $1000 \mathrm{mg}$ metformin for 6 months had a significantly greater decline in glucose, body mass index and HbA1c levels [34]. In our study, we took metformin for only 2 weeks. It may not be effective on the human unless taking several months as this study. However, we worried that taking metformin for a longer time and delaying surgery might not be feasible in actual clinical settings, we decided the 2 weeks periods from diagnosis until surgery. We consider that the safety of taking $1000 \mathrm{mg}$ metformin in non-DM patients has been proved, we think that we can increase the internal dose of metformin $(1000 \mathrm{mg} / \mathrm{day})$. In addition, there are not established evaluation methods on immune factors. In particular, $\mathrm{H}$-score is not popular because the method of calculation is confusing.
If taking metformin has some influences on the immune system, it would be possible to improve or achieve a synergistic effect on systemic therapy like chemotherapy, hormone and any targeting therapies. And if the effects of metformin on immune function require longer treatment to manifest, we should be continued postoperatively or be combined with adjuvant therapies. Nicole et al. showed that combination of metformin with PD-1 blockade improved intra-tumoral T-cell function and tumor clearance. As a result of modifications of tumor microenvironment, there might be some possibility to release immunotherapy resistance [8]. At present, there are ongoing clinical trials including the following: "A Phase III Randomized Trial of Metformin vs Placebo in Early Stage Breast Cancer (NCT01101438)", "A Study of Liposomal Doxorubicin + Docetaxel + Trastuzumab + Metformin in Operable and Locally Advanced HER2 Positive Breast Cancer (NCT02488564)", "Neoadjuvant Toremifene With Melatonin or Metformin in Locally Advanced Breast Cancer", and "Metformin Plus Neoadjuvant Chemotherapy in Breast Cancer". Our study did not show a statistically significant difference, there were some tendency that metformin increases or has some effects on the immune functions.

\section{Conclusion}

The TIL values in CNB specimens are a reliable indicator of the TIL status of the entire resected breast tumor.

Preoperative metformin tends to increase TILs, CD4 +, $\mathrm{CD} 8+$ lymphocytes and IFN $\gamma$, suggesting enhancement of immunological anti-tumor response in the patients with breast cancer.

Acknowledgements This report was supported in part by the National Cancer Center Research and Development Fund (29-A-3) from the Ministry of Health, Labour and Welfare and the Practical Research for Innovative Cancer Control (19ck0106307h0003) from Japan Agency for Medical Research and Development, AMED.

Funding This study was not funded.

\section{Compliance with ethical standards}

Conflict of interest All authors have not received research grants from any Company and there is no non-financial conflict of interest.

Ethical approval All procedures performed in studies involving human participants were in accordance with the ethical standards of the institutional and/or national research committee and with the 1964 Helsinki declaration and its later amendments or comparable ethical standards.

Informed consent Informed consent was obtained from all individual participants included in the study. 
Open Access This article is licensed under a Creative Commons Attribution 4.0 International License, which permits use, sharing, adaptation, distribution and reproduction in any medium or format, as long as you give appropriate credit to the original author(s) and the source, provide a link to the Creative Commons licence, and indicate if changes were made. The images or other third party material in this article are included in the article's Creative Commons licence, unless indicated otherwise in a credit line to the material. If material is not included in the article's Creative Commons licence and your intended use is not permitted by statutory regulation or exceeds the permitted use, you will need to obtain permission directly from the copyright holder. To view a copy of this licence, visit http://creativecommons.org/licenses/by/4.0/.

\section{References}

1. Perou CM, Sorlie T, Eisen MB, van de Rijin M, Jeffrey SS, Rees CA, Pollack JR, Ross DT, Johnsen H, Akslen LA et al (2000) Molecular portraits of human breast tumours. Nature 406:747-752

2. Sotiriou C, Pusztai L (2009) Gene-expression signatures in breast cancer. N Engl J Med 360(8):790-800

3. Zhou Y, Zhang X, Gu C, Xia J (2015) Diabetes mellitus is associated with breast cancer: systematic review, meta-analysis, and in silico reproduction. Panminerva Med 57(3):101-108

4. Chen L, Chubak J, Boudreau DM, Barlow WE, Weiss NS, Li CI (2017) Diabetes treatments and risks of adverse breast cancer outcomes among early-stage breast cancer patients: a SEER-medicare analysis. Cancer Res 77(21):6033-6041

5. Barbieri F, Thellung S, Ratto A, Carra E, Marini V, Fucile C, Bajetto A, Pattarozzi A, Würth R, Gatti M et al (2015) In vitro and in vivo antiproliferative activity of metformin on stem-like cells isolated from spontaneous canine mammary carcinomas: translational implications for human tumors. BMC Cancer 15:228

6. Hirsch HA, Iliopoulos D, Tsichlis PN, Struhl K (2009) Metformin selectively targets cancer stem cells and acts together with chemotherapy to block tumor growth and prolong remission. Cancer Res 69:7507-7511

7. Eikawa S, Nishida M, Mizukami S, Yamazaki C, Nakayama E, Udono H (2015) Immune-mediated antitumor effect by type 2 diabetes drug, metformin. Proc Natl Acad Sci USA 112(6):1809-1814

8. Nicole E Scharping, Ashley V Menk, Ryan D Whetstone, Xue Zeng and Greg M Delgoffe: Efficacy of PD-1 Blockade is Potentiated by Metformin-Induced Reduction of Tumor Hypoxia. Cancer Immunol Res Jan;5(1): 9-16, 2017

9. Gu-Trantien C, Loi S, Garaud S, Equeter C, Libin M, de Wind A, Ravoet M, Le Buanec H, Sibille C, Manfouo-Foutsop G et al (2013) CD4(+) follicular helper T cell infiltration predicts breast cancer survival. J Clin Invest 123:2873-2892

10. Romagnani S (1997) The Th1/Th2 paradigm. Immunol Today 18(6):263-266

11. Mao Y, Qu Q, Zhang Y, Liu J, Chen X, Shen K (2014) The value of tumor infiltrating lymphocytes (TILs) for predicting response to neoadjuvant chemotherapy in breast cancer: a systematic review and meta-analysis. PLOS One 9(12):e115103

12. Hwang HW, Jung H, Hyeon J, Park YH, Ahn JS, Im YH, Nam SJ, Kim SW, Lee JE, Yu JH et al (2019) A nomogram to predict pathologic complete response ( $\mathrm{pCR}$ ) and the value of tumor-infiltrating lymphocytes (TILs) for prediction of response to neoadjuvant chemotherapy (NAC) in breast cancer patients. Breast Cancer Res Treat 173(2):255-266

13. Ono M, Tsuda H, Shimizu C, Yamamoto S, Shibata T, Yamamoto H, Hirata T, Yonemori K, Ando M, Tamura K et al (2012) Tumor-infiltrating lymphocytes are correlated with response to neoadjuvant chemotherapy in triple-negative breast cancer. Breast Cancer Res Treat 132(3):793-805

14. Denkert C, von Minckwitz G, Brase JC, Sinn BV, Gade S, Kronenwett R, Pfitzner BM, Salat C, Loi S, Schmitt WD et al (2015) Tumor-infiltrating lymphocytes and response to neoadjuvant chemotherapy with or without carboplatin in human epidermal growth factor receptor 2-positive and triple-negative primary breast cancer. J Clin Oncol 33(9):983-991

15. Mao Y, Qu Q, Chen X, Huang O, Wu J, Shen K (2016) The prognostic value of tumor-infiltrating lymphocytes in breast cancer: a systematic review and meta-analysis. PLOS One 11(4).

16. Rathore AS, Kumar S, Konwar R, Makker A, Negi MP, Goel MM (2014) CD3+, CD4+ \& CD8+ tumour infiltrating lymphocytes (TILs) are predictors of favourable survival outcome in infiltrating ductal carcinoma of breast. Indian J Med Res 140(3):361-369

17. Schmid P, Adams S, Rugo HS, Schneeweiss A, Barrios CH, Iwata H, Diéras V, Hegg R, Im SA, Shaw Wright G et al (2018) Atezolizumab and nab-paclitaxel in advanced triple-negative breast cancer. N Engl J Med 379(22):2108-2121

18. Denkert C, Wienert S, Poterie A, Loibl S, Budczies J, Badve S, Bago-Horvath Z, Bane A, Bedri S, Brock J et al (2016) Standardized evaluation of tumor-infiltrating lymphocytes in breast cancer: results of the ring studies of the international immuno-oncology biomarker working group. Mod Pathol 29(10):1155-1164

19. Denkert C, Loibl S, Noske A, Roller M, Müller BM, Komor M, Budczies J, Darb-Esfahani S, Kronenwett R, Hanusch C et al (2010) Tumor-associated lymphocytes as an independent predictor of response to neoadjuvant chemotherapy in breast cancer. $\mathrm{J}$ Clin Oncol 28(1):105-113

20. Loi S, Paish EC, Powe DG, Macmillan RD, Grainge MJ, Lee AH, Ellis IO, Green AR (2013) Prognostic and predictive value of tumor infiltrating lymphocytes in a phase III randomized adjuvant breast cancer trial in node-positive breast cancer comparing the addition of docetaxel to doxorubicin with doxorubicin-based chemotherapy: BIG 02-98. J Clin Oncol 31(7):860-867

21. Mahmoud SM, Paish EC, Powe DG, Macmillan RD, Grainge MJ, Lee AH, Ellis IO, Green AR (2011) Tumor-infiltrating CD8+ lymphocytes predict clinical outcome in breast cancer. J Clin Oncol 29(15):1949-1955

22. Kanda Y (2013) Investigation of the freely available easy-to-use software 'EZR' for medical statistics. Bone Marrow Transplant 48:452-458

23. Salgado R, Denkert C, Demaria S, Sirtaine N, Klauschen F, Pruneri G, Wienert S, Van den Eynden G, Baehner FL, PenaultLlorca $\mathrm{F}$ et al (2015) The evaluation of tumor infiltrating lymphocytes (TILs) in breast cancer: recommendations by an International TILs Working Group 2014. Ann Oncol 26(2):259-271

24. Cha YJ, Ahn SG, Bae SJ, Yoon CI, Seo J, Jung WH, Son EJ, Jeong J (2018) Comparison of tumor-infiltrating lymphocytes of breast cancer in core needle biopsies and resected specimens: a retrospective analysis. Breast Cancer Res Treat 171(2):295-302

25. Jones RL, Salter J, A'Hern R, Nerurkar A, Parton M, Reis-Filho JS, Smith IE, Dowsett M (2010) Relationship between oestrogen receptor status and proliferation in predicting response and longterm outcome to neoadjuvant chemotherapy for breast cancer. Breast Cancer Res Treat 119(2):315-323

26. Hadad S, Iwamoto T, Jordan L, Purdie C, Bray S, Baker L, Jellema G, Deharo S, Hardie DG, Pusztai L et al (2011) Evidence for biological effects of metformin in operable breast cancer: a pre-operative, window-of-opportunity, randomized trial. Breast Cancer Res Treat 128(3):783-794

27. Macaskill EJ, Bartlett JM, Sabine VS, Faratian D, Renshaw L, White S, Campbell FM, Young O, Williams L, Thomas JS et al (2011) The mammalian target of rapamycin inhibitor everolimus (RAD001) in early breast cancer: results of a pre-operative study. Breast Cancer Res Treat 128(3):725-734 
28. Hrushesky WJ, Retsky M, Baum M, Demicheli R (2007) Prognostic value of Ki67 expression after short term presurgical endocrine therapy for primary breast cancer - Response. J Natl Cancer Inst 99(13):1053-1054

29. Ellis MJ, Coop A, Singh B, Tao Y, Llombart-Cussac A, Jänicke F, Mauriac L, Quebe-Fehling E, Chaudri-Ross HA, Evans DB et al (2003) Letrozole inhibits tumor proliferation more effectively than tamoxifen independent of HER $1 / 2$ expression status. Cancer Res 63(19):6523-6531

30. Baselga J, Semiglazov V, van Dam P, Manikhas A, Bellet M, Mayordomo J, Campone M, Kubista E, Greil R, Bianchi G et al (2009) Phase II randomized study of neoadjuvant everolimus plus letrozole compared with placebo plus letrozole in patients with estrogen receptor-positive breast cancer. J Clin Oncol 27(16):2630-2637

31. Viale G, Giobbie-Hurder A, Regan MM, Coates AS, Mastropasqua MG, Dell'Orto P, Maiorano E, MacGrogan G, Braye SG, Ohlschlegel C et al (2008) Prognostic and predictive value of centrally reviewed Ki-67 labeling index in postmenopausal women with endocrine-responsive breast cancer: results from Breast International Group Trial 1-98 comparing adjuvant tamoxifen with letrozole. J Clin Oncol 26(34):5569-5575

32. Jones RL, Salter J, A'Hern R, Nerurkar A, Parton M, Reis-Filho JS, Smith IE, Dowsett M (2009) The prognostic significance of Ki67 before and after neoadjuvant chemotherapy in breast cancer. Breast Cancer Res Treat 116(1):53-68

33. Wherry EJ (2011) T cell exhaustion. Nat Immunol 12(6):492-499

34. Ko KP, Ma SH, Yang JJ, Hwang Y, Ahn C, Cho YM, Noh DY, Park BJ, Han W, Park SK (2015) Metformin intervention in obese non-diabetic patients with breast cancer : phase II randomized, double-blind, placebo-controlled trial. Breast Cancer Res Treat 153(2):361-370

Publisher's Note Springer Nature remains neutral with regard to jurisdictional claims in published maps and institutional affiliations. 